\title{
A fully microscopical simulation of nuclear collisions by a new QMD model
}

\author{
Maria Vittoria Garzelli ${ }^{\mathrm{a}}$ \\ University of Milano, Department of Physics, and INFN, Sezione di Milano, 20133 Milano, Italy
}

\begin{abstract}
Nucleon-ion and ion-ion collisions at non relativistic bombarding energies can be described by means of Monte Carlo approaches, such as those based on the Quantum Molecular Dynamics (QMD) model. We have developed a QMD code, to simulate the fast stage of heavy-ion reactions, and we have coupled it to the de-excitation module available in the FLUKA Monte Carlo transport and interaction code. The results presented in this work span the projectile bombarding energy range within $200-600 \mathrm{MeV} / \mathrm{A}$, allowing to investigate the capabilities and limits of our non-relativistic QMD approach.
\end{abstract}

\section{General framework and motivation of this work}

A fully microscopical simulation of nucleon-ion and ionion collisions, at nucleon level, can be performed, among several different approaches [1], by means of Quantum Molecular Dynamics (QMD) models [2]. They are dynamical models which allow to study the phase-space evolution of the projectile-target colliding systems, from their initial mutual trajectory influence and eventually their overlap, depending on the impact parameter, to the compression phase, accompanied by a temperature and density increase, up to the following expansion stage, characterized by the formation of hot excited fragments (pre-fragments). The whole phase occurs on a time scale within a few hundreds $\mathrm{fm} / \mathrm{c}$, depending on the size of the colliding systems and the bombarding energy, and is called the "fast" stage of the reaction.

Additionally, improved versions of QMD models (e.g., the CoMD one, developed by Papa et al. [3]) allow to compute the system evolution even for a longer time, up to thousands $\mathrm{fm} / \mathrm{c}$, and thus have also been used to describe pre-fragment de-excitation, at least in its initial stage. This has led to direct comparisons of the results of improved QMD simulations to experimental data concerning fragment emission distributions. On the other hand, pre-fragment de-excitation can occur on a time scale even larger (up to $\sim 10^{-15} \mathrm{~s}$ ). Thus, a complete treatment of this slow stage can be covered by different models, generally based on statistical considerations. The underlying assumption in applying one of these statistical models to nuclear systems is that they are thermalized. While at the lowest energies the colliding ions stay close to each other for a time long enough for thermalization to occur, to define a temperature for the whole system at higher energies (several tens $\mathrm{MeV} / \mathrm{A}$ ) can be very problematic, since the expansion phase can begin before a global thermalization process is completed. Anyway, in the last case, at advanced time in the expansion stage pre-fragments are well separated and each of them is supposed to be thermalized. Whereas theoretical models allow to compute an excitation energy for each pre-fragment, from the experimental point of view the problem of the determination of hot fragment temperatures

${ }^{a}$ Presenting author, e-mail: maria.garzelli@mi.infn.it

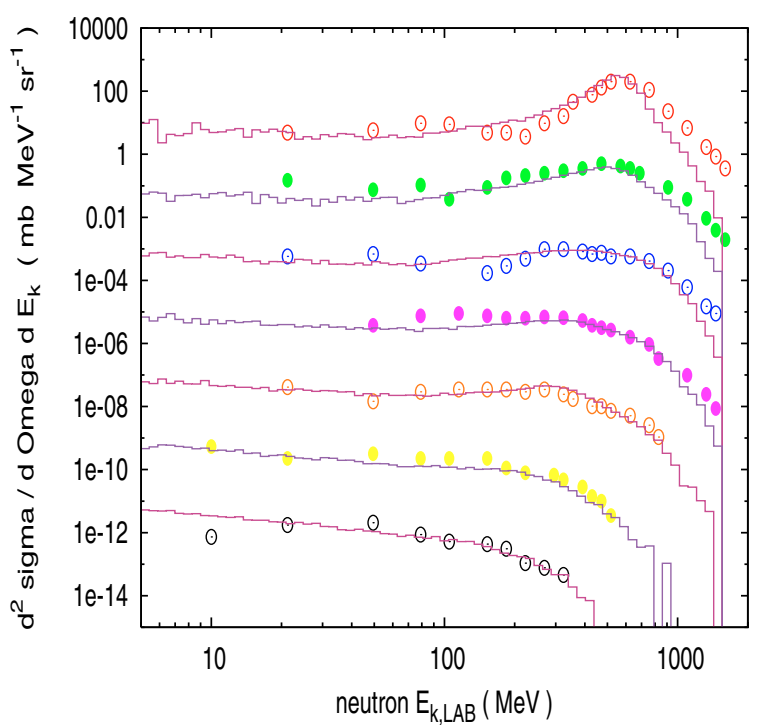

Fig. 1. Double-differential neutron production cross section for Ar projectiles impinging on $\mathrm{C}$ at $560 \mathrm{MeV} / \mathrm{A}$ bombarding energy. The results of the theoretical simulations made by QMD + FLUKA deexcitation are shown by solid histograms, while the experimental data taken from ref. [14] are shown by circles. Distributions at $5^{\circ}, 10^{\circ}$, $20^{\circ}, 30^{\circ}, 40^{\circ}, 60^{\circ}$ and $80^{\circ}$ (lab) angles are scaled by decreasing even powers of 10 .

is still open. On the other hand, planned applications (such as hadrontherapy and space radioprotection) need models and tools to calculate doses to human bodies and equipment, due to radiation exposure. In particular, reliable calculations of physical doses require an accurate description of nuclear interactions. Nuclear reaction models used for predictions in these applications, should be capable of reproducing available experimental data concerning particle and fragment emission.

This paper presents the results of simulations performed with a newly developed QMD code, coupled to the de-excitation module available in one of the most widely used Monte Carlo transport and interaction codes, the FLUKA code [4,5]. In particular, FLUKA includes algorithms allowing to compute evaporation, fission, fragmentation and Fermi break-up (for light nuclei) followed by $\gamma$ emission, for 


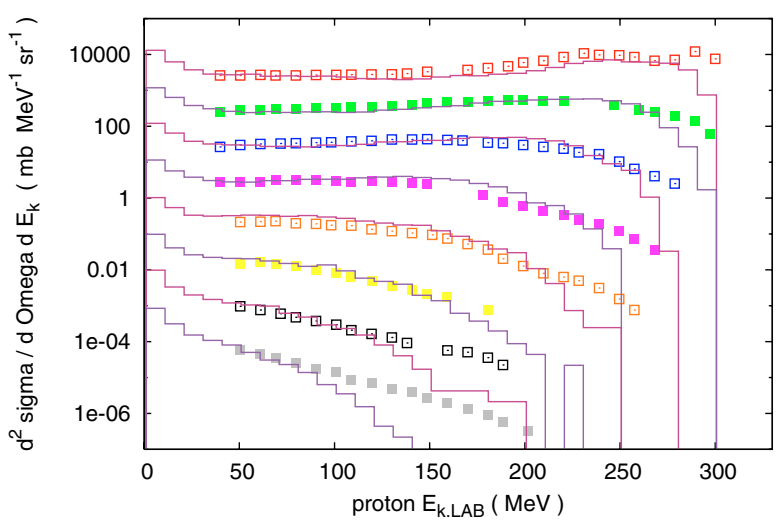

Fig. 2. Double-differential proton production cross section for $p$ projectiles impinging on $\mathrm{C}$ at $300 \mathrm{MeV} / \mathrm{A}$ bombarding energy. The results of the theoretical simulations are shown by solid histograms, while the experimental data from ref. [15] are shown by squares. Distributions at $20^{\circ}, 30^{\circ}, 40^{\circ}, 50^{\circ}, 60^{\circ}, 75^{\circ}, 90^{\circ}$ and $105^{\circ}(\mathrm{lab})$ angles are scaled by $10^{4}, 10^{3}, 10^{2}, 10^{1}, 10^{0}, 10^{-1}, 10^{-2}, 10^{-3}$, respectively.

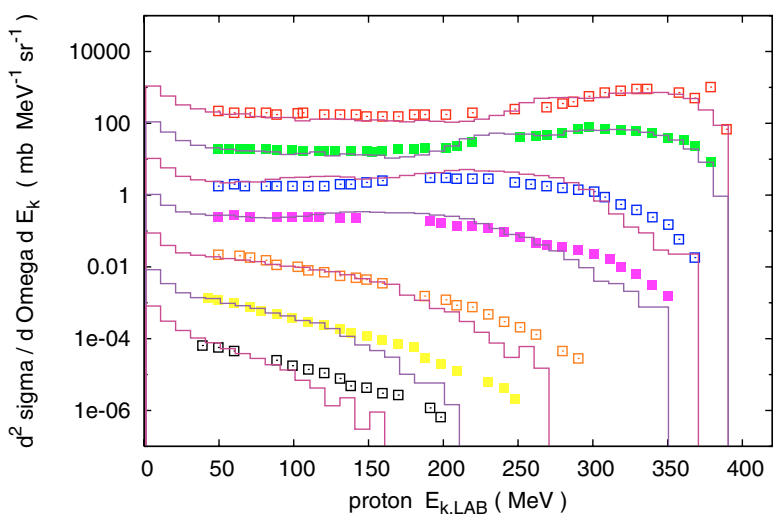

Fig. 3. The same as figure 2 for $\mathrm{p}$ impinging on $\mathrm{C}$ at $392 \mathrm{MeV}$. Distributions at $20^{\circ}, 25^{\circ}, 40^{\circ}, 50^{\circ}, 75^{\circ}, 90^{\circ}$ and $105^{\circ}$ (lab) angles are scaled by $10^{3}, 10^{2}, 10^{1}, 10^{0}, 10^{-1}, 10^{-2}, 10^{-3}$, respectively.

whichever excited pre-fragment. Those algorithms are part of the FLUKA general hadron-nucleus (and lepton-nucleus) interaction model called PEANUT (PreEquilibrium Approach to NUclear Thermalization) [6]. For further information, the interested reader can check also refs. [7-10] and the FLUKA website http://www.fluka.org. The excited pre-fragments computed by the QMD model at the end of the fast stage are then passed through these algorithms for the final de-excitation and fragment generation.

\section{Comparisons with experimental data}

\subsection{Neutron emission}

As far as neutron emission is concerned, performances of the model in predicting double-differential neutron production cross sections in the projectile energy range from several tens $\mathrm{MeV} / \mathrm{A}$ up to $400 \mathrm{MeV} / \mathrm{A}$ have already been shown in refs. [11-13]. The QMD we have developed so far includes non-relativistic potentials, thus it is expected to work at
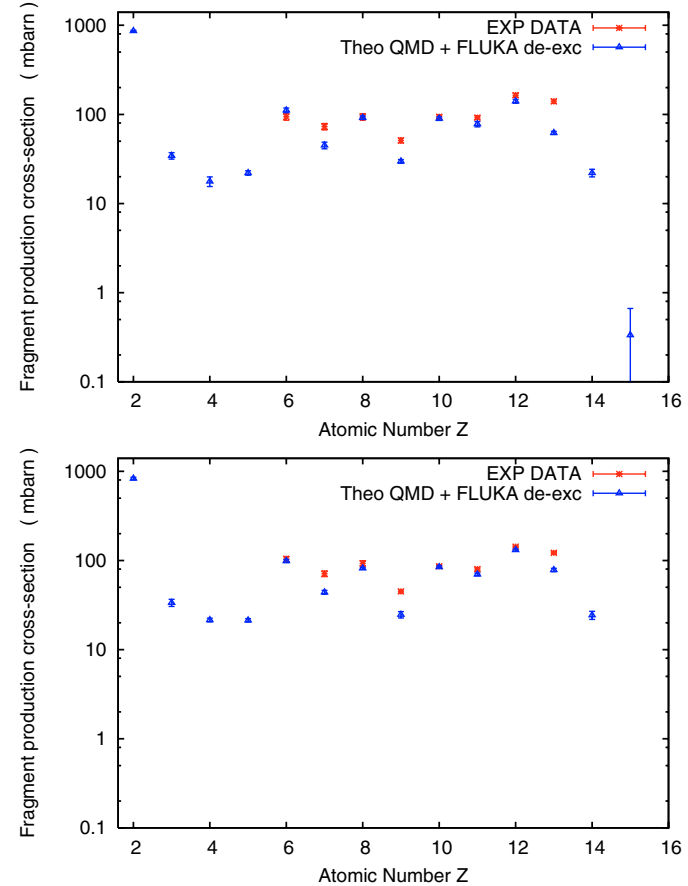

Fig. 4. Charged projectile-like fragment production cross sections for $\mathrm{Si}$ ions impinging on $\mathrm{C}$ at 266 and $344 \mathrm{MeV} / \mathrm{A}$ bombarding energies, respectively in the upper and in the lower panel: theoretical predictions are compared to experimental data from ref. [16]. Detector acceptance has been included in the theoretical simulations.

energies up to a few hundreds $\mathrm{MeV} / \mathrm{A}$, limited also by the inability to deal with pion production and reinteraction. In the effort of better understanding up to which energies it can be reasonably applied, and which modifications have to be included to improve it, a few simulations at energies above $\sim 500 \mathrm{MeV} / \mathrm{A}$ have also been performed. As an example, the neutron double-differential production cross section for $\mathrm{Ar}+$ $\mathrm{C}$ at $560 \mathrm{MeV} / \mathrm{A}$ bombarding energy is shown in figure 1 . The experimental data taken from ref. [14] are plotted together with the theoretical curves. It is apparent that, as far as forward emission angles are concerned $\left(5-10^{\circ}\right)$, the model underestimates neutron emission tails from $\sim 800 \mathrm{MeV} / \mathrm{A}$ up to the highest energies. On the other hand, at $>20^{\circ}$ emission angles, the agreement of the theoretical results with the experimental data is quite encouraging, and at angles $\geq 40^{\circ}$ the neutron tails are nicely reproduced.

\subsection{Proton emission}

While systematic data concerning neutron emission in heavyion collisions exist, as far as proton emission in ion-ion collisions is concerned, less data are available in literature. We thus start considering nucleon induced reactions, for which many more data concerning proton emission exist. Indication of the performances of our code in reproducing the experimental data presented by [15] is shown in figure 2 and 3 for $\mathrm{p}+\mathrm{C}$ at 300 and $392 \mathrm{MeV}$ bombarding energies, respectively. The linear scale on the energy axis allows to better appreciate the position of the broad emission peaks. With the exception of 

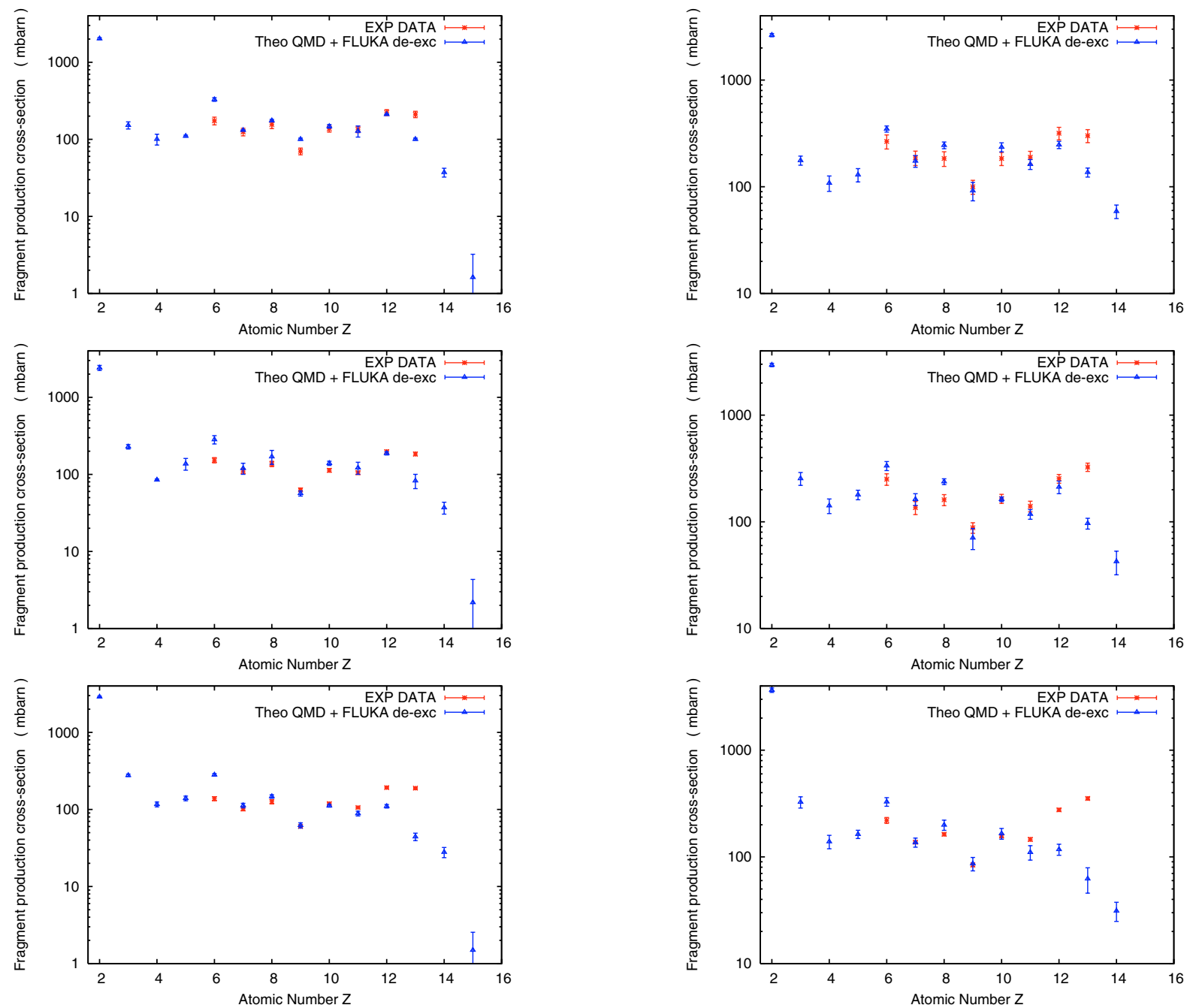

Fig. 5. The same as figure 4 for $\mathrm{Si}$ ions impinging on $\mathrm{Cu}$ at 274 , 344 and $545 \mathrm{MeV} / \mathrm{A}$ bombarding energies, respectively in the upper, intermediate and lower panel.

the quasi-elastic peak, the general agreement is quite good as far as forward emission angles are concerned, while the neutron tails at larger angles are underestimated, especially at the highest energies. The slight departure from smoothness of some of the lines obtained by simulation is not due to statistics. It is instead an artifact introduced by the fact that quite a few QMD ion initial states have been used to perform these simulations, where projectiles are single protons. In fact, only seven ${ }^{12} \mathrm{C}$ initial states have been used to obtain these figures. As a general result, when nucleon - ion or light ion light ion simulations are performed, better results are obtained considering a larger number of QMD initial states.

\subsection{Projectile-like fragment emission}

As far as fragment emission is concerned, a few results obtained by our QMD coupled to FLUKA de-excitation have already been presented in refs. $[12,13]$.

In the present paper, the focus is given to the case of projectile-like fragments produced by $\mathrm{Si}$ ions impinging on

Fig. 6. The same as figure 4 for $\mathrm{Si}$ ions impinging on $\mathrm{Pb}$ at 274 , 364 and $540 \mathrm{MeV} / \mathrm{A}$ bombarding energies, respectively in the upper, intermediate and lower panel.

targets made of $\mathrm{C}, \mathrm{Cu}$ and $\mathrm{Pb}$. Systematic studies of $\mathrm{Si}$ fragmentation on targets of light, intermediate and heavy composition have been carried out in the last few years at the HIMAC in Chiba, and at the BNL AGS. The results of these experiments were recently published [16]. Comparisons with the predictions of our model, as far as projectile-like fragment production cross sections are concerned, are under way. A few results, for the lowest energies cases, are plotted in figures 4, 5,6. Detector acceptance was taken into account in performing our simulations by means of cuts in energy and angle, which allow to select only projectile-like fragments escaping the target within a few degrees around forward direction, according to the angular acceptances given in [16]. Due to the features of the detectors used in these experiments, data are available for fragments with charge $Z=6-13$ only. For lower charges $(Z \leq 5)$, it was very difficult to disentangle the peaks corresponding to the separate contribution of each fragment species to the energy released in the Si detectors used. Sometime, also the broad peaks corresponding to the $\mathrm{Z}=6$, 7 charges were determined with some uncertainties, especially 


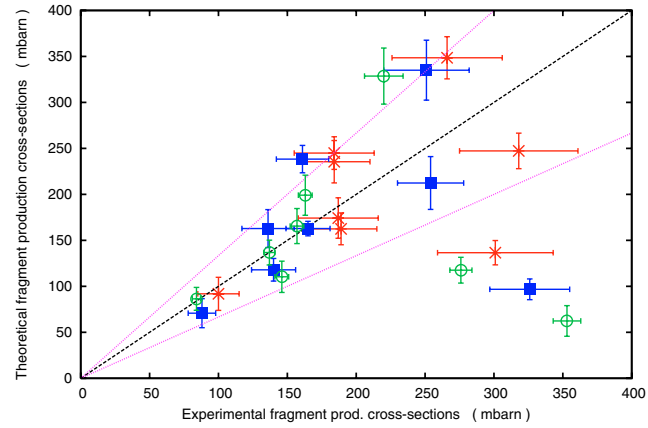

Fig. 7. Scatterplot of fragment production cross sections predicted by QMD + FLUKA de-excitation (vertical axis) vs. measured cross sections (horizontal axis) for $\mathrm{Si}$ ions impinging on $\mathrm{Pb}$ at 274 (crosses), 364 (squares) and 540 (circles) MeV/A bombarding enegies. The errorbars on the theoretical results are statistical only. The two lines respectively above and below the diagonal correspond to deviations of the modelled results from the experimental ones by $\pm 33 \%$. The largest discrepancies are seen for the $\mathrm{Z}=13$ fragment production cross sections.

at the lowest energies. Anyway, the authors of ref. [16] observe that their largest uncertainties concern the determination of the $\mathrm{Z}=13$ fragment production cross sections, due to superposition effects with the high-energy tails of primary ions that cross the targets without interacting, which have to be cut. These uncertainties are more important at lower energies, due to the use of thin targets to avoid significant energy losses of projectiles, which would lead to misleading reaction cross sections.

In all cases discussed in the present paper, the theoretical model systematically underestimates the $Z=13$ cross sections, while for $\mathrm{Cu}$ and $\mathrm{Pb}$ targets it overestimates the $\mathrm{Z}=6$ production cross sections. The best agreement with the experimental data is instead observed for fragments with $Z=10,11$, and for fragments with $Z=12$ at energies below $400 \mathrm{MeV} / \mathrm{A}$. At higher energies, an underestimation of the $Z=12$ fragment abundances occurs as well. This could be an indication that the QMD developed so far gives more reliable results when used in the study of central collisions, while it is more difficult to apply it to the study of stripping reactions, where only one or two nucleons of the projectile ion interact with the target. Furthermore, the authors of ref. [16] suggest the hyphotesis that electromagnetic dissociation can contribute to the $\mathrm{Z}=12,13$ fragment production cross sections at high energy for the heaviest targets. At present, we have not tested this hypothesis yet. Finally, as far as Fluorine fragments $(Z=9)$ are concerned, in all cases the theoretical model confirms their suppression, at least from a qualitative point of view, with respect to the abundance of the other species close in charge, as observed in the experiment.

To obtain a global overview of the deviations of the predictions of our theoretical model from the experiments, the results can be summarized in scatterplots, such as the one shown in figure 7 for the $\mathrm{Si}+\mathrm{Pb}$ case, including data at different energies for fragments with $Z=6-13$. With the exception of the cases of the heaviest (and lightest) charged fragments already discussed, the figure does not show particular systematics. For the heavy target considered, it is apparent that the discrepancies between the model predictions and the experimental data do not exceed $\sim 30-35 \%$ in most cases. Theoretical fragmentation models based on QMD codes provide, in general, more reliable predictions in case of intermediate and heavy mass targets than in case of lighter ones.

Collaboration with F. Ballarini, G. Battistoni, F. Cerutti, A. Fassò, A. Ferrari, E. Gadioli, A. Ottolenghi, L.S. Pinsky, J. Ranft and P.R. Sala is acknowledeged. This work was supported by the University of Milano.

\section{References}

1. A. Boudard (these proceedings).

2. J. Aichelin, Phys. Rep. 202, 233 (1991).

3. M. Papa, T. Maruyama, A. Bonasera, Phys. Rev. C 64, 024612$1,6(2002)$.

4. A. Fassò, A. Ferrari, S. Roesler et al., Proc. Computing in High Energy and Nuclear Physics 2003 Conference (CHEP2003), La Jolla, CA, USA, March 24-28, 2003 (paper MOMT005) eConf C0303241, (2003) hep-ph/0306267.

5. A. Fassò, A. Ferrari, J. Ranft, P.R. Sala, CERN Yellow Report 2005-10, INFN/TC_05/11, 1-387 (2005).

6. A. Ferrari, P.R. Sala, Proc. Workshop on Nuclear Reaction Data and Nuclear Reactors Physics, Design and Safety, Trieste, Italy, April 1996, edited by A. Gandini, G. Reffo (1998), pp. 2-424.

7. F. Ballarini, G. Battistoni, F. Cerutti et al., talk presented at ND2004, Santa Fe, NM, US, Sept. 26-Oct. 1 2004, AIP Conf. Proc. 769, (2005), pp. 1197-1202.

8. G. Battistoni, F. Cerutti, R. Engel et al., Proc. $11^{\text {th }}$ International Conference on Nuclear Reaction Mechanisms, Varenna, Italy, June 12-16 2006, edited by E. Gadioli, Ricerca Scientifica ed Educazione Permanente, Suppl. 126, Univ. Milano (2006), pp. 483-495.

9. A. Ferrari, P.R. Sala, J. Ranft, S. Roesler, Z. Phys. C 71, 75 (1996).

10. A. Ferrari, P.R. Sala, J. Ranft, S. Roesler, Z. Phys. C 70, 413 (1996).

11. M.V. Garzelli, F. Ballarini, G. Battistoni et al., Proc. $11^{\text {th }}$ International Conference on Nuclear Reaction Mechanisms, Varenna, Italy, June 12-16 2006, edited by E. Gadioli, Ricerca Scientifica ed Educazione Permanente, Suppl. 126, Univ. Milano (2006), pp. 515-524, nucl-th/0610018.

12. M.V. Garzelli, P.R. Sala, G. Battistoni et al., Proc. $25^{\text {th }}$ Workshop on Nuclear Theory, June 26-July 1 2006, edited by Rila Mountains, Bulgaria, S. Dimitrova, Diomira Press, Sofia (2006), pp. 123-131, nucl-th/0610050.

13. M.V. Garzelli, P.R. Sala, F. Ballarini et al. (submitted to Adv. Space Res.), nucl-th/0611075.

14. Y. Iwata, T. Murakami, H. Sato et al., Phys. Rev. C 64, 0546091,10 (2001).

15. T. Kin, F. Saiho, S. Hohara et al., Phys. Rev. C 72, 014606-1,9 (2005).

16. C. Zeitlin, A. Fukumura, S.B. Guetersloh et al., Nucl. Phys. A 784, 341 (2007). 